\title{
A CNN-based Cell Tracking Method for Multi-Slice Intravital Imaging Data
}

\author{
Kenji Fujimoto \\ Tsubasa Mizugaki \\ Department of Bioinformatic \\ Engineering, Graduate School of \\ Information Science and Technology, \\ Osaka University \\ Suita, Japan \\ Yutaka Uchida \\ Masaru Ishii \\ Department of Immunology and Cell \\ Biology, Graduate School of Medicine, \\ Osaka University \\ Suita, Japan
}

\author{
Utkrisht Rajkumar \\ Department of Computer Science and \\ Engineering, University of California \\ San Diego, CA, USA
}
Vineet Bafna
Department of Computer Science and Engineering, University of California
San Diego, CA, USA

\author{
Hironori Shigeta \\ Shigeto Seno \\ Department of Bioinformatic \\ Engineering, Graduate School of \\ Information Science and Technology, \\ Osaka University \\ Suita, Japan \\ Hideo Matsuda \\ Department of Bioinformatic \\ Engineering, Graduate School of \\ Information Science and Technology, \\ Osaka University \\ Suita, Japan \\ matsuda@ist.osaka-u.ac.jp
}

\begin{abstract}
Cell migration is one of the important criteria for determining effects on cells by inflammatory and/or chemical stimulation. Accurate detection of cells' movement through traditional methods, such as optical flow, is difficult because those cells' fluorescence intensity and shapes are similar to each other. Therefore, we adopt a tracking approach based on a convolutional neural network (CNN) using time-lapse multi-slice images observed with 2-photon excitation microscopy. Existing CNN-based cell tracking methods are often focusing on tracking targets in the 2-dimensional (2D) space since the costs for computation and annotation drastically increase in 3-dimensional (3D) settings. Those methods usually convert the 3D microscopic images to the $2 \mathrm{D}$ ones via maximum intensity projection (MIP). However, as MIP does not keep the depth information, it is difficult to track depth-directionally overlapping cells in MIP images accurately. To cope with the problem, we propose a novel CNN-based cell tracking method for multi-slice 3D images. Our method trains a CNN using MIP images annotated with the cell locations, similarly to the existing methods. Meanwhile, in the tracking phase, our method estimates not only each cell's location but its depth using multiple slices at different depths. Using our method, we track leukocyte migration in multi-slice time-lapse images observed with 2-photon excitation microscopy. As a result, we show that our method outperforms existing cell tracking methods including multi-domain network (MDNet) with MIP images.
\end{abstract}

Permission to make digital or hard copies of all or part of this work for personal or classroom use is granted without fee provided that copies are not made or distributed for profit or commercial advantage and that copies bear this notice and the full citation on the first page. Copyrights for components of this work owned by others than ACM must be honored. Abstracting with credit is permitted. To copy otherwise, or republish, to post on servers or to redistribute to lists, requires prior specific permission and/or a fee. Request permissions from permissions@acm.org.

BCB '21, August 1-4, 2021, Gainesville, FL, USA

(C) 2021 Association for Computing Machinery.

ACM ISBN 978-1-4503-8450-6/21/08 . .\$15.00

https://doi.org/10.1145/3459930.3469559

\section{CCS CONCEPTS}

- Computing methodologies $\rightarrow$ Tracking; • Applied computing $\rightarrow$ Imaging.

\section{KEYWORDS}

cell tracking, microscopy imaging, deep learning, computational biology

ACM Reference Format:

Kenji Fujimoto, Tsubasa Mizugaki, Utkrisht Rajkumar, Hironori Shigeta, Shigeto Seno, Yutaka Uchida, Masaru Ishii, Vineet Bafna, and Hideo Matsuda. 2021. A CNN-based Cell Tracking Method for Multi-Slice Intravital Imaging Data. In 12th ACM International Conference on Bioinformatics, Computational Biology and Health Informatics (BCB '21), August 1-4, 2021, Gainesville, FL, USA. ACM, New York, NY, USA, 7 pages. https://doi.org/10.1145/3459930. 3469559

\section{INTRODUCTION}

Cell migration is an essential process during many phases of development. Also in adult life, it continues to play an extremely important role in processes such as wound healing and the functioning of the immune system [3]. Understanding movement of single cells and cells in tissues requires the analysis of the complex processes under normal and perturbed conditions. This typically includes the trajectory analysis of large numbers of migrating cells in microscopic videos, i.e., time-lapse image sequences.

Live imaging of dynamic processes at the cellular level has been made possible by the development of fluorescent biomarkers and rapid advances in optical microscopy technology. Especially, intravital microscopy allows observing biological processes in live animals at a high resolution that makes distinguishing between individual cells of a tissue possible [13]. The data produced through intravital microscopy are typically multi-slice 3-dimensional (3D) images, which consist of multiple 2-dimensional (2D) images called slices at different depths. We show an example of such data in Figure 1. Figure (a) is the 3D view of leukocytes (neutrophils, green) in a 


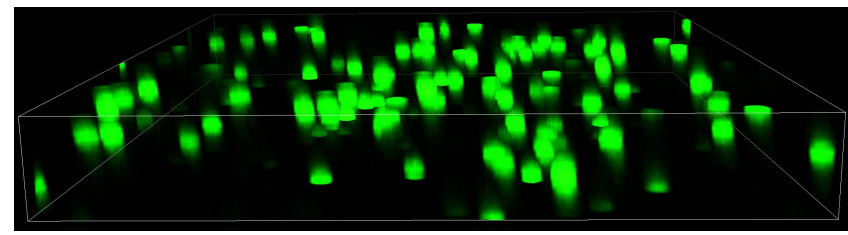

(a) $3 \mathrm{D}$ view.

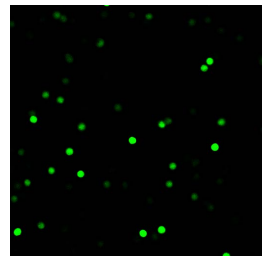

(b) Slice at $z=1$

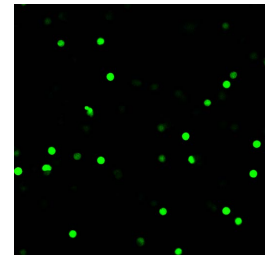

(c) Slice at $z=10$.
Figure 1: An image of leukocytes (green) obtained through 2-photon excitation microscopy. The resolution of this image is $512(\mathrm{X}$, horizontal $) \times 512(\mathrm{Y}$, vertical $) \times 15(\mathrm{Z}$, depth $)$. The image (a) is visualized by Nikon NIS-Elements Viewer software.

collagen gel medium observed with 2-photon excitation microscopy. Figure (b) and (c) are slices of that image at different depths.

In order to extract migrating cells' trajectories from such videos, cell tracking [10], i.e., estimating each cell's location over frames, is a fundamental task. Various cell tracking methods have been proposed up to the present. Most of them are individually adjusted to the specific cell types and/or experimental conditions.

Meanwhile, in the field of general object tracking, convolutional neural networks (CNNs) are gathering attentions. They are capable of adapting to the specific features of the targets by learning from training data, i.e., videos annotated with the target locations (ground-truth). Therefore, they do not require manual adjustment. This characteristic is also useful in cell tracking, where the targeted cell's appearance and/or dynamics widely vary according to the experimental conditions. In fact, some recent cell tracking methods $[6-9,16]$ have incorporated CNNs.

However, existing CNN-based cell tracking methods mainly focus on phase-contrast or bright-field 2D images. We consider that simply extending them to the 3D space for multi-slice 3D data may not be a practical idea. This is because the costs for computation and annotation drastically increase in 3D settings. In terms of the computational cost, compared to a $2 \mathrm{D}$ video, a multi-slice $3 \mathrm{D}$ video whose frames are composed of $d$ slices may require $d$ times larger memory consumption and longer operation time. Annotation means manual cell tracking in order to produce the ground-truth. In one of the most common settings, each desired target location is represented as a cuboid covering the cell of interest, called a bounding box. In that case, to annotate a 3D video with ground-truth, the annotator must determine the $\mathrm{X}$ coordinate, $\mathrm{Y}$ coordinate, $\mathrm{Z}$ coordinate, width, height, and depth of the bounding box for all targets and frames, while checking 3D images like Figure 1. Therefore, as the numbers of frames and/or cells increase, it becomes

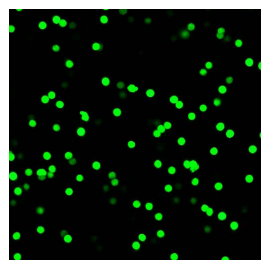

Figure 2: The MIP image of Figure 1.

almost impossible to accurately annotate 3D videos. A common approach to cope with the above difficulties is to convert multi-slice $3 \mathrm{D}$ images to $2 \mathrm{D}$ ones via maximum intensity projection (MIP), and perform tracking in the $2 \mathrm{D}$ space. In $2 \mathrm{D}$ settings, each bounding box is a rectangle covering the target, i.e., a 4-tuple of its $\mathrm{X}$ coordinate, $\mathrm{Y}$ coordinate, width, and height. The MIP image of Figure 1 is shown in Figure 2 . The intensity $i_{\mathrm{MIP}}(x, y)$ of the MIP image at $(x, y)$ of the MIP image is defined as

$$
i_{\mathrm{MIP}}(x, y)=\max _{z} i(x, y, z),
$$

where $i(x, y, z)$ denotes the intensity of the original multi-slice 3D image at $(x, y, z)$. However, as MIP does not keep the depth information, depth-directionally overlapping cells are not separated in MIP images. Then, even for CNN-based methods, accurately tracking such cells is extremely difficult because those cells' fluorescence intensity and shapes are similar to each other as shown in Figure 2.

To address the above problem, we propose a novel CNN-based cell tracking method for multi-slice imaging data. Our method is the extended version of an object tracking method based on multidomain network (MDNet) [12]. It is a CNN originally proposed for general object tracking and has been confirmed to be robust against illumination variation, shape variation, and background clutter, which are also common difficulties in cell tracking. To accurately track depth-directionally overlapping cells, our method estimates not only each target's location but its depth simultaneously. Specifically, in the tracking phase, our method explores the target from several slices around the estimated depth of the previous frame. Therefore, our method is robust to targeted cells' depth-directional overlap, compared to existing methods using MIP videos. As our method utilizes a few slices for tracking, not the whole 3D images, its computational cost is not as much as the cost of $3 \mathrm{D}$ tracking. In addition, our method uses the MIP videos in its training phase, similarly to existing CNN-based methods. Therefore, our method requires the annotation cost similar to existing methods. In this paper, we apply our method to the multi-slice $3 \mathrm{D}$ videos of leukocyte migration and compare the performance of our method with those of existing methods.

The rest of this paper is organized as follows. Section 2 introduces existing cell tracking methods. Section 3 presents our CNN-based cell tracking method. Section 4 shows the results of the performance comparison. Section 5 discusses the results for furthur improvement of our method. Section 6 concludes this paper.

\section{EXISTING METHODS}

Cell tracking is a fundamental task for extracting cells' migration trajectories from microscopic videos, and various methods have 


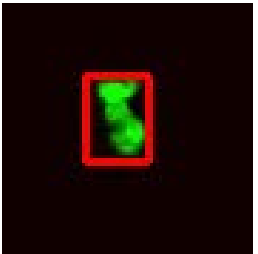

(a) Previous frame

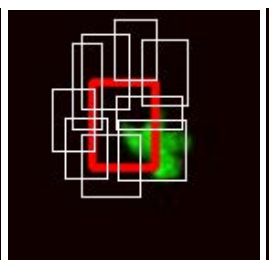

(b) Sampling.

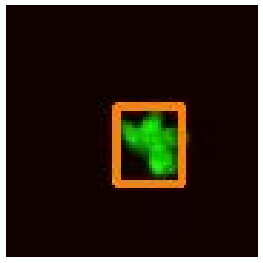

(c) Location estimation
Figure 3: MDNet-based tracking. The red, white, and orange rectangles represent the target's previous location, a sampled bounding box, the bounding box with the highest score (i.e., the estimated location), respectively. The original images are from the dataset utilized in our experiments. See 4.1 for details.

been proposed up to the present. Most of them are based on segmentation and association. They perform segmentation (i.e., recognizing the target's regions from an image) to detect all cells in each frame and associate the detected cell regions over frames. For example, LineageTracker [4] associates detected cells based on their similarities of the apperance features. Also, various other approaches including particle filters [5], mean shift clustering [18], and image matching [15] have been adopted for cell tracking. Because those methods are designed for the specific cell types and/or experimental conditions, they require manual adjustment against the individual dataset.

Meanwhile, approaches based on deep learning, especially convolutional neural networks $(\mathrm{CNN})$, are recently gathering attention in the filed of target tracking. In CNN-based tracking methods, CNNs, i.e., mathematical models for tracking, is trained (optimized) to fit the specific data, that are called training data and composed of videos with the ground-truth annotation. Therefore, they do not require manual adjustment, and also are achieving remarkable performance in both general object tracking $[2,12]$ and cell tracking [6-9, 16]. Among them, multi-domain network (MDNet) [12] is a CNN for tracking a single target in the first frame of the input video. MDNet-based tracking is illustrated in Figure 3. It finds the target in order from the first frame. For each frame, it samples multiple bounding boxes around the previous location (Figure 3 (b)) and calculates the score of each bounding box. That score represents the possibility of the sampled bounding box being the target's location and is calculated by MDNet. Then, the bounding box with the highest score is chosen as the target location in that frame (Figure 3 (c)). MDNet is not only trained in prior to tracking, but updated during tracking to adapt the specific target's features. See the original paper [12] for details. As far as we know, MDNet has not been utilized for cell tracking yet. However, it has been confirmed to be robust against difficult situations in object tracking such as illumination variation, shape variation, and background clutter [12], that are also common in cell tracking. Therefore, we argue that MDNet is also effective for cell tracking, and we extend it for multi-slice intravital imaging data in the next section.

\section{PROPOSED METHOD}

\subsection{Overview}

In this section, we propose the extended MDNet-based tracking method for intravital imaging data. In order to tackle cells' depthdirectional overlap, our method simultaneously estimates each cell's location (bounding box) and depth, as shown in Figure 4. Specifically, for each frame, it explores the target from slices around the estimated depth of the previous frame. Because cells are located sparsely in slices compared to MIP images as shown in Figure 2, our method accurately tracks even depth-directionally overlapping cells. In addition, the computational cost of our method is much smaller than that of 3D tracking since it explores targets from a few slices, not the whole 3D image.

In prior to tracking, our method trains MDNet following the original training procedure. The training data are MIP images with ground-truth annotation, similarly to existing methods. Therefore, the costs for annotation and computation required in the training phase is similar to ones required by existing methods using MIP images.

\subsection{Tracking}

3.2.1 Preliminaries. Here we define the notation utilized in the following explanation. Each frame of the input multi-slice $3 \mathrm{D}$ video is denoted by $I_{1}, \cdots, I_{T}$, where $T$ is the total number of frames. $I_{t}(\mathbf{x}, d)$ represents the region of the $d$-th slice of $I_{t}$ cropped according to the bounding box $\mathbf{x}$. The $c$-th cell's estimated bounding box and estimated depth in the $t$-th frame are denoted as $\mathbf{x}_{t}^{(c)}=\left(x_{t}^{(c)}, y_{t}^{(c)}, w_{t}^{(c)}, h_{t}^{(c)}\right)$ and $d_{t}^{(c)}$, respectively. Here $x, y, w$, and $h$ represent the X coordinate of the left end, Y coordinate of the top end, width, and height of the rectangle, respectively. In addition, we define $f$ as a function that takes an image as input and returns the score of the input image in $[0,1]$ calculated by MDNet.

3.2.2 Initial segmentation. MDNet is originally a model for tracking a single object in the first frame of a video, and assumes that the initial target location (bounding box) is given as input. Therefore, our method clarifies all targets' initial locations in prior to MDNet-based tracking. To this end, our method performs an arbitrary segmentation algorithm for the initial MIP frame. Then, for each segmented region (cell) $c=1, \cdots, m$, our method estimates the $c$-th cell's initial location $\mathbf{x}_{1}^{(c)}$ as the bounding box of the $c$-th segmented region.

3.2.3 Estimation of the initial depth. For each cell $c=1, \cdots, m$, our method estimates its initial depth $d_{1}^{(c)}$ as follows. First, our method calculates the score of the initial location $\mathbf{x}_{1}^{(c)}$ at all depths. Then, it chooses the depth with the highest score as the estimated initial depth $d_{1}^{(c)}$.

$$
d_{1}^{(c)}=\arg \max _{d} f\left(I_{1}\left(\mathbf{x}_{1}^{(c)}, d\right)\right) .
$$

3.2.4 Estimation of the location and depth in each frame. For each frame $I_{t}(2 \leq t \leq T)$, our method estimates the location of each cell $c, \mathbf{x}_{t}^{(c)}$, and depth $d_{t}^{(c)}$ based on those of the previous frame, $\mathbf{x}_{t-1}^{(c)}$ and $d_{t-1}^{(c)}$, as shown in Figure 4 . Note that Figure 4 illustrates 


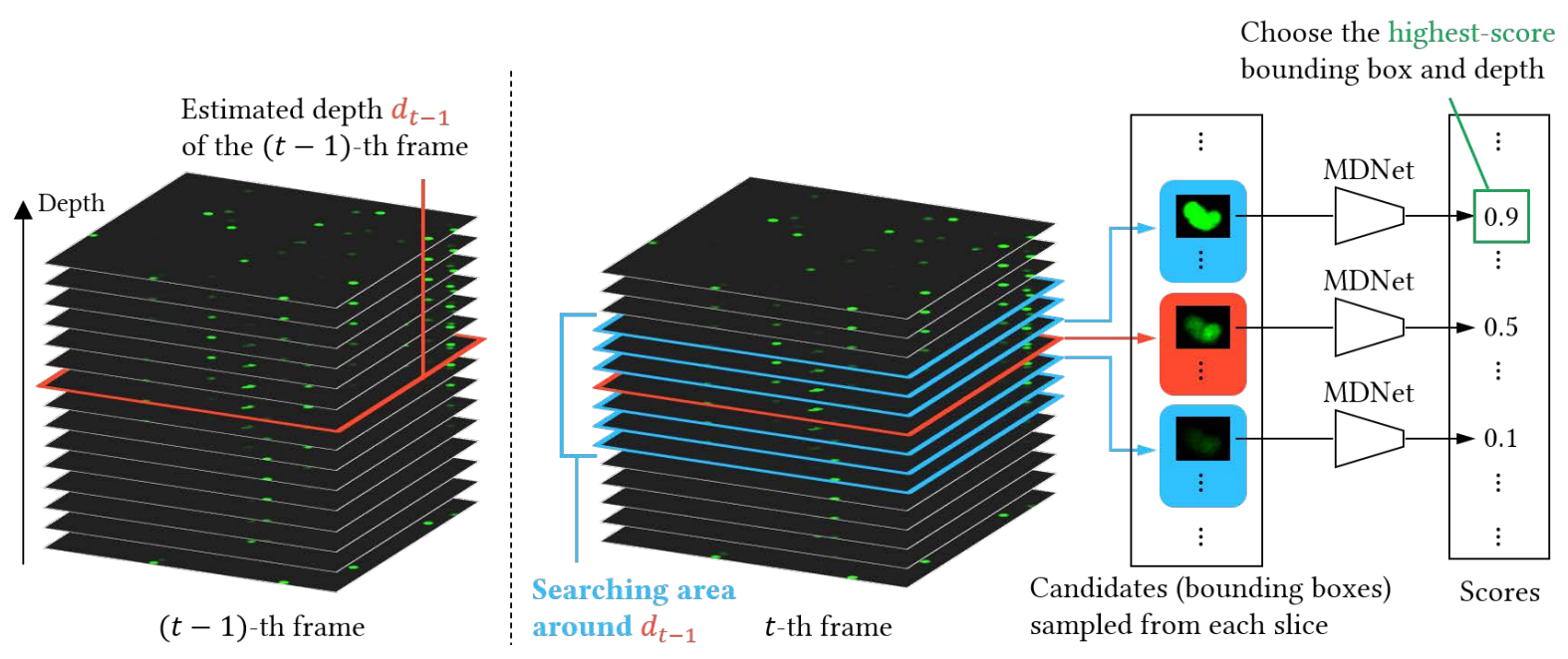

Figure 4: Overview of our cell tracking method. In each frame, our method explores the target from slices around the estimated depth of the previous frame. Although this figure only illustrates the procedure for one individual target for the sake of simplicity, our method also estimates the other targets' locations and depths similarly.

the estimation of one individual cell for the sake of simplicity and omits the cell's identifier $c$.

First, as performed by the original MDNet-based tracking method, our method samples a certain number $(N)$ of bounding boxes $\mathbf{x}_{t}^{(c, 1)}, \cdots, \mathbf{x}_{t}^{(c, N)}$ around the previous location $\mathbf{x}_{t-1}^{(c)}$. They are the candidates for the current bounding box $\mathbf{x}_{t}^{(c)}$. Specifically, each candidate $\mathbf{x}_{t}^{(c, i)}=\left(x_{t}^{(c, i)}, y_{t}^{(c, i)}, w_{t}^{(c, i)}, h_{t}^{(c, i)}\right)$ is generated as follows.

$$
\begin{gathered}
x_{t}^{(c, i)} \sim G\left(x_{t-1}^{(c)},\left(a_{1} r\right)^{2}\right), \\
y_{t}^{(c, i)} \sim G\left(y_{t-1}^{(c)},\left(a_{1} r\right)^{2}\right), \\
w_{t}^{(c, i)}=w_{1}^{(c)} \cdot a_{2}{ }^{s_{i}}, \\
h_{t}^{(c, i)}=h_{1}^{(c)} \cdot a_{2}{ }^{s_{i}},
\end{gathered}
$$

where $G\left(\mu, \sigma^{2}\right)$ denotes a Gaussian distribution, $r$ is the average of the previous width and height, $s_{i} \sim G\left(a_{3}, a_{4}\right)$, and $a_{1}, \ldots, a_{4}$ are fixed hyperparameters. Equation 3 and 4 indicate that our sampling is based on the assumption that the target's movement speed in the X-Y plane depends on its size (more precisely, the average of its previous width and height).

Second, our method samples the candidates for the current depth $d_{t}^{(c)}$. We assume that each cell's depth-directional movement speed is similar to the one in the X-Y plane. Therefore, our method samples all integers in the $95 \%$ confidence interval of the Gaussian distribution $G\left(d_{t-1}^{(c)},\left(a_{1} r\right)^{2}\right)$. The $j$-th candidate is as follows.

$$
d_{t}^{(c, j)}=d_{t-1}^{(c)}-\left\lfloor 2 a_{1} r \frac{d_{2}}{d_{1}}\right\rfloor+(j-1),
$$

where $d_{1}$ and $d_{2}$ are the pixel sizes in the X-Y plane and depthdirection, respectively.
From the candidates, our method chooses the bounding box and depth with the highest score as the current ones.

$$
\left(\mathbf{x}_{t}^{(c)}, d_{t}^{(c)}\right)=\left(\mathbf{x}_{t}^{\left(c, i^{\prime}\right)}, d_{t}^{\left(c, j^{\prime}\right)}\right)
$$

where

$$
\left(i^{\prime}, j^{\prime}\right)=\arg \max _{(i, j)} f\left(I_{t}\left(\mathbf{x}_{t}^{(c, i)}, d_{t}^{(c, j)}\right)\right) .
$$

As performed by the original MDNet-based tracking method, our method also performs the bounding box regression (BBR), which is a procedure to modify estimated bounding boxes to fit the target more precisely, and online fine-tuning, during the above procedures.

\subsection{Training}

For training MDNet in prior to tracking, we propose to utilize the MIP videos with ground-truth annotation as training data, instead of slices. As shown in Figure 2, the number of cells in MIP images is much larger than slices. Therefore, we consider MIP videos contain more cells that are difficult to track accurately, and are more effective for training. Furthermore, we propose to train MDNet only with data of cells whose movement is sufficiently large. In typical intravital imaging data, the majority of cells remain almost stationary from the initial frame to the final frame of the video. However, because tracking cells with larger motion is more difficult, we consider the percentage of such cells in the training data should not be small. Therefore, our method chooses cells whose longest inter-frame movement is larger than the threshold $d$ and trains the MDNet only with those data.

Our training procedure is basically as conducted for the original MDNet. One difference is regarding the final layer of the network. Since the training procedure for original MDNet aims to track objects belonging to various domains, it switches the final layer (namely, the domain-specific layer) according to the target's domain. In contrast, because this study does not desire to track various types of objects, we do not incorporate that mechanism. 


\section{EXPERIMENTAL RESULTS}

We applied our method to the intravital imaging dataset and compared its tracking performance with existing methods. One of the utilized existing methods is the original MDNet-based tracking using MIP videos. Hereinafter, we refer to this method as MDNet-MIP. The other method is LineageTracker (referred to as LT), a cell tracking method based on segmentation and association. Although there are other CNN-based cell tracking methods $[6-9,16]$, we do not compare our method with them. This is because cells are visualized as light spots and have inadequate visual features in fluorescent images, compared to phase-contrast or bright-field images, on which existing CNN-based methods are mainly focusing.

\subsection{Data}

We utilized 13 time-lapse multi-slice images visualizing leukocytes in the collagen gel medium obtained through 2-photon excitation microscopy. In 2 of the data, the leukocytes were stimulated with the lipopolysaccharide (LPS). In the other 11 data, the leukocytes were stimulated with the granulocyte-macrophage colony-stimulating factor (GM-CSF). The videos with the LPS stimulation were composed of 91 frames. In terms of the data with the GM-CSF stimulation, 2 videos were composed of 91 frames, and the other 9 videos were composed of 61 frames. All videos were taken at 1 minute intervals. For each video, the resolution on the X-Y plane was 512 $\times 512$ pixels, and 1 pixel was equivalent to $0.5 \mu \mathrm{m}$. In terms of the depth-direction, 15 slices were taken at $3.0 \mu \mathrm{m}$ intervals.

\subsection{Implementation}

We basically followed the original paper of MDNet [12] for settings of the network architecture and hyperparameters. Specifically, hyperparameters in Equation 3-6 were set as $a_{1}=0.3, a_{2}=0.25$, $a_{3}=0, a_{4}=0.25$. The MDNet models utilized in our method and MDNet-MIP are identical, including trainable parameters. For the initial segmentation, we utilized a graph-cut-based algorithm [19] We implemented our method and MDNet-MIP using Python and PyTorch, a deep learning framework. For these methods, we divided the whole dataset into 5 datasets, which contained 2 or 3 videos respectively, and conducted 5-fold cross validation. Meanwhile, for LineageTracker, we utilized the plugin with the same name [4] of ImageJ [14]. The parameters of LineageTracker were set to their default values. All experiments were conducted on a computer with i9-10980XE 3.00GHz CPU, 64GB RAM, and Quadro RTX 8000 GPU.

\subsection{Metrics}

We evaluated the tracking performance using the metrics summarized in Table 1. All these metrics are commonly used in various competitions of general object tracking such as MOT17 [11] False-positive (FP), false-negative (FN), identity switches (IDSW), multiple object tracking accuracy (MOTA), and multiple object tracking precision (MOTP) have been proposed as CLEAR MOT metrics [1]. These metrics are based on the matching procedure between ground-truth trajectories and estimated trajectories (tracking results), which is illustrated in Figure 5. In each frame, each ground-truth object is matched with an estimated target location (hereinafter, referred to as detection) if the intersection-over-union (IoU) of the ground-truth object and detection is equal to or above
Table 1: Metrics for evaluating tracking performance

\begin{tabular}{|c|c|c|c|}
\hline Metric & Better & Perfect & Description \\
\hline $\mathrm{FP}$ & lower & 0 & $\begin{array}{l}\text { Number of false-positives (misde- } \\
\text { tections) over all frames. }\end{array}$ \\
\hline FN & lower & 0 & $\begin{array}{l}\text { Number of false-negatives (unde- } \\
\text { tected ground-truth targets) over } \\
\text { all frames. }\end{array}$ \\
\hline IDSW & lower & 0 & $\begin{array}{l}\text { Number of trajectory switches for } \\
\text { the same objects. }\end{array}$ \\
\hline MOTA & higher & 1 & $\begin{array}{l}\text { Overall tracking accuracy. See } \\
\text { Equation } 10 .\end{array}$ \\
\hline MOTP & higher & 1 & $\begin{array}{l}\text { Mean IoU (described in } 4.3 \text { ) over all } \\
\text { true-positive detections. }\end{array}$ \\
\hline MT & higher & 1 & $\begin{array}{l}\text { Rate of ground-truth trajectories } \\
\text { tracked for } 80 \% \text { of their lifespans } \\
\text { or more. }\end{array}$ \\
\hline ML & lower & 0 & $\begin{array}{l}\text { Rate of ground-truth trajectories } \\
\text { tracked for } 20 \% \text { of their lifespans } \\
\text { or less. }\end{array}$ \\
\hline
\end{tabular}

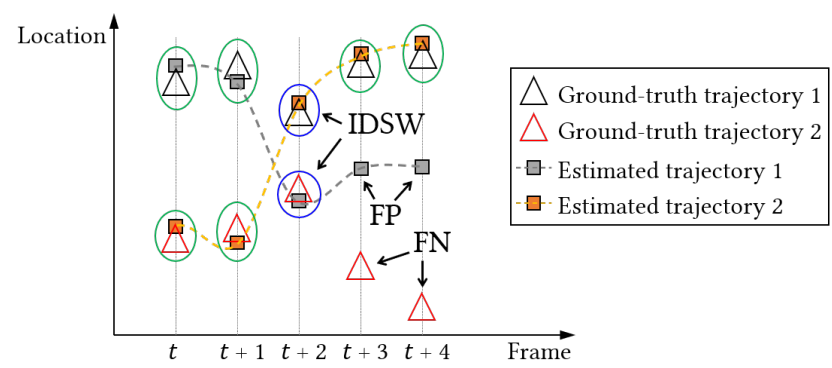

Figure 5: Matching procedures in CLEAR MOT metrics. Each triangle denotes a ground-truth object, and each rectangle with a dashed line denotes an estimated location. The different colors distinguish the identities. Each ellipse represents a frame-wise matching between a ground-truth object and an estimated location.

the threshold. The IoU of two regions is defined as the ratio of the area of their overlapping (intersection) region to the area of their union region, and it is 1 when the two regions are identical, while it is 0 when the two regions do not overlap. If the IoU is below the threshold, the ground-truth object is not matched with any detections. In other words, the ground-truth object is not detected, and thus that error is counted among FN. In contrast, if a detection is not matched with any ground-truth object, that error is counted among FP. Such errors are shown at the $(t+3)$-th frame and $(t+4)$-th frame in Figure 5. In addition, at the $(t+2)$-th frame, each ground-truth object corresponding the detected identity has changed from the previous frames. The situation means that each tracker has mistaken its target, and thus such a error is counted among IDSW. See the original paper [1] for details. In our experiment, we set the IoU threshold as 0.3. The definition of MOTA is as 
follows.

$$
\text { MOTA }=1-\frac{F P+F N+\text { IDSW }}{\text { GT }},
$$

where GT denotes the sum of the numbers of ground-truth objects in each frame, and the other symbols are as defined in Table 1.

We also utilized the metrics mostly-tracked (MT) [17] and mostlylost (ML) [17] to evaluate how many frames the targets are tracked since tracking each cell in as many consequtive frames as possible is important to analyze cell dynamics.

\subsection{Results}

Table 2 shows the performance comparison. For most of the metrics, our method achieved the best mean performance. Remarkably, IDSW (number of trajectory swiches for the same targetts), MT (rate of mostly-tracked targets), and ML (rate of mostly-lost targets) of our method were better than those of MDNet-MIP. These results suggest that our method succeeded in tracking individual cells continuously for more frames without trajectory switches, compared to existing methods. Such ability is extremely important for the purpose of understanding the movement of individual cells. In contrast, existing methods outperformed our method in terms of FP and MOTP. We consider the causes and future improvement plan about these issues in Section 5.

Figure 6 shows examples of tracking results of our method compared with those of MDNet-MIP. As shown in the 3D views in the second row, the targeted leukocyte is passing under another leukocyte. In MIP images, the two leukocytes were overlapping and hardly distinguishable visually, as shown in the first row. Then, MDNet-MIP mistook the other leukocyte as its tracking target. On the other hand, our method was tracking the targeted leukocyte continuously because it estimated the target's location and depth simultaneously to distinguish the overlapping targets correctly.

\section{DISCUSSION AND FUTURE WORK}

Although our experimental results show the effectiveness of our method for most of the metrics, FP and MOTP of our method were not better than those of existing method. Here we discuss the causes and improvement plans about these issues.

We consider that one reason for the higher FP of our method and MDNet-MIP was the existence of cells tracked by multiple different trajectories. These two methods can be regarded as operating multiple MDNet-based tracker in pararell. In such a situation, if a tracker mistakes the other tracker's target as its own, that target is tracked by two trackers. MDNet-MIP easily causes this kind of errors because it is not able to distinguish depth-directionally overlapping cells. Even with our method, if multiple cells' both locations and depths are sufficiently close, such a error may still happen. In the computation of CLEAR MOT metrics, each ground-truth object is matched with at most one detection (estimated location), while the other detections are regarded as false-positives. In contrast, LineageTracker never causes such errors because its approach is based on segmentation and association. Our future plan to solve this problem is to make closely-located trackers interact and modify their trajectories when their targets' collision is detected.

In terms of MOTP, we consider that the difference in fluorescence intensity between MIP images and slices affected the results. Figure 7 shows an identical cell in a MIP image and two slices. The
MIP image visualizes the entire cell clearly, while some regions are not clear in the slices. That phenomenon is natural because each intensity of the MIP image is the maximum one of all-depth slices. As our method explores the target from slices, the estimated bounding boxes may be smaller than ones estimated from MIP images. We consider that this is the reason why MOTP of our method (i.e., the mean IoU of all true-positive detections) was lower than that of MDNet-MIP. This issue may cause problems for the purpose such as temporal observation of cell sizes. Our idea to resolve it is to improve the BBR module, which makes estimated bounding boxes fit the target more precisely. It is currently based on a single slice. We consider utilizing slices at different depths and/or the MIP image may help improve the performance of our method.

\section{CONCLUSION}

In this paper, we proposed a method for tracking cell migration in multi-slice intravital imaging data. Our method is an extended version of MDNet-based object tracking method and tracks depthdirectionally overlapping cells more accurately via slice-based estimation of target locations and depths. Our experiments to track leukocyte migration in multi-slice images showed that it outperformed existing methods including MDNet with MIP images.

\section{ACKNOWLEDGMENTS}

This work was supported in part by JST CREST Grant Number JPMJCR15G1; JSPS KAKENHI Grant Numbers JP18H04124, JP19K22894, and JP20H04947, Japan.

\section{REFERENCES}

[1] Keni Bernardin and Rainer Stiefelhagen. 2008. Evaluating Multiple Object Tracking Performance: The CLEAR MOT Metrics. EURASIP fournal on Image and Video Processing 2008 (2008), 1-10.

[2] Gioele Ciaparrone, Francisco Luque Sánchez, Siham Tabik, Luigi Troiano, Roberto Tagliaferri, and Francisco Herrera. 2020. Deep learning in video multi-object tracking: A survey. Neurocomputing 381 (2020), 61-88.

[3] Dirk Dormann and Cornelis J. Weijer. 2006. Imaging of cell migration. EMBO fournal 25, 15 (2006), 3480-3493.

[4] Mike J. Downey, Danuta M. Jeziorska, Sascha Ott, T. Katherine Tamai, Georgy Koentges, Keith W. Vance, and Till Bretschneider. 2011. Extracting fluorescent reporter time courses of cell lineages from high-throughput microscopy at low temporal resolution. PloS one 6, 12 (2011), e27886.

[5] Kenji Fujimoto, Shigeto Seno, Hironori Shigeta, Tomohiro Mashita, Masaru Ishii, and Hideo Matsuda. 2020. Tracking and Analysis of FUCCI-Labeled Cells Based on Particle Filters and Time-to-Event Analysis. International fournal of Bioscience, Biochemistry and Bioinformatics 10, 2 (2020), 94-109.

[6] Junya Hayashida and Ryoma Bise. 2019. Cell Tracking with Deep Learning for Cell Detection and Motion Estimation in Low-Frame-Rate. In Medical Image Computing and Computer Assisted Intervention - MICCAI 2019. Springer International Publishing, 397-405.

[7] David E. Hernandez, Steven W. Chen, Elizabeth E. Hunter, Edward B. Steager, and Vijay Kumar. 2018. Cell Tracking with Deep Learning and the Viterbi Algorithm. In 2018 International Conference on Manipulation, Automation and Robotics at Small Scales (MARSS). 1-6. https://doi.org/10.1109/MARSS.2018.8481231

[8] Haigen Hu, Lili Zhou, Qiu Guan, Qianwei Zhou, and Shengyong Chen. 2018. An Automatic Tracking Method for Multiple Cells Based on Multi-Feature Fusion. IEEE Access 6 (2018), 69782-69793. https://doi.org/10.1109/ACCESS.2018.2880563

[9] Jean-Baptiste Lugagne, Haonan Lin, and Mary J. Dunlop. 2020. DeLTA: Automated cell segmentation, tracking, and lineage reconstruction using deep learning. PLOS Computational Biology 16, 4 (04 2020), 1-18.

[10] Erik Meijering, Oleh Dzyubachyk, and Ihor Smal. 2012. Methods for Cell and Particle Tracking. In Imaging and Spectroscopic Analysis of Living Cells. Methods in Enzymology, Vol. 504. Academic Press, 183-200.

[11] Anton Milan, Laura Leal-Taixé, Ian Reid, Stefan. Roth, and Konrad Schindler. 2016. MOT16: A Benchmark for Multi-Object Tracking. arXiv:1603.00831 [cs] (2016). http://arxiv.org/abs/1603.00831 arXiv: 1603.00831. 
Table 2: Performance comparison. Each result is written in the format of mean \pm standard deviation. Higher and lower values are better for the metrics with the symbols $\uparrow$ and $\downarrow$, respectively. The best mean for each metric is written in bold.

\begin{tabular}{l|c|c|c|c|c|c|c}
\hline \multicolumn{1}{c|}{ Method } & FP $\downarrow$ & FN $\downarrow$ & IDSW $\downarrow$ & MOTA $\uparrow$ & MOTP $\uparrow$ & MT $\uparrow$ & ML $\downarrow$ \\
\hline LT & $\mathbf{1 1 0 . 7 6 9} \pm 90.926$ & $695.231 \pm 893.758$ & $3.538 \pm 4.069$ & $0.537 \pm 0.179$ & $0.524 \pm 0.050$ & $0.476 \pm 0.181$ & $0.187 \pm 0.102$ \\
MDNet-MIP & $127.154 \pm 117.639$ & $127.154 \pm 117.639$ & $5.077 \pm 8.695$ & $0.792 \pm 0.154$ & $\mathbf{0 . 7 0 7} \pm 0.045$ & $0.876 \pm 0.077$ & $0.014 \pm 0.023$ \\
Ours & $111.077 \pm 88.061$ & $\mathbf{1 1 1 . 0 7 7} \pm 88.061$ & $\mathbf{3 . 1 5 4} \pm 4.588$ & $\mathbf{0 . 8 2 6} \pm 0.111$ & $0.686 \pm 0.047$ & $\mathbf{0 . 9 0 3} \pm 0.059$ & $\mathbf{0 . 0 0 8} \pm 0.016$ \\
\hline
\end{tabular}
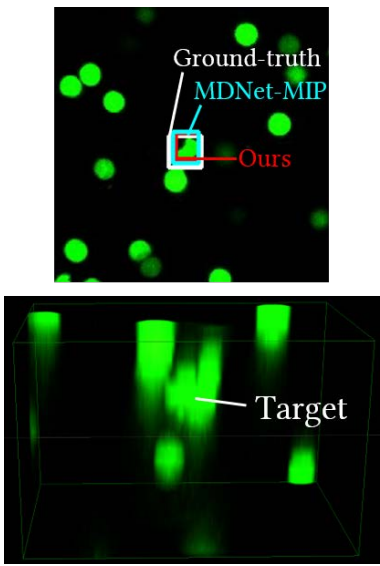

(a) 35th frame
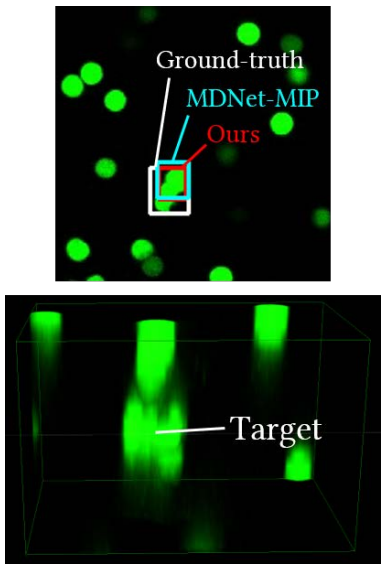

(b) 44th frame
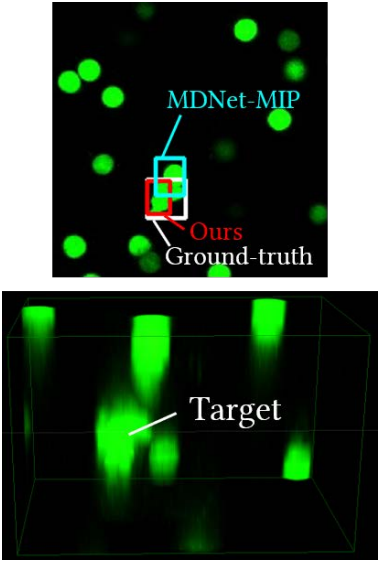

(c) 47 th frame
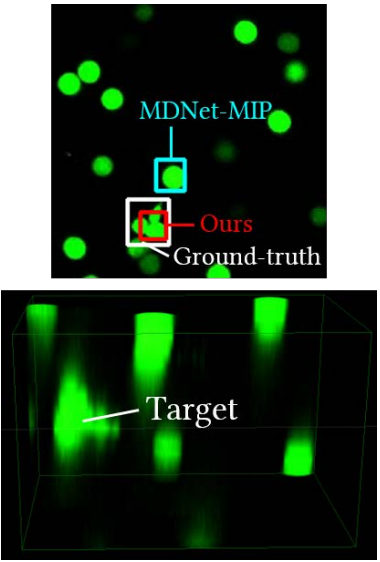

(d) 55th frame

Figure 6: Examples of the tracking results. In the first row, ground-truth bounding boxes (white), estimated bounding boxes of our method (red), and MDNet-MIP (light blue) are shown on the MIP images. Images in the second row are respectively the $3 \mathrm{D}$ views corresponding to the above image. All images are cropped to ensure visibility.



(a) MIP

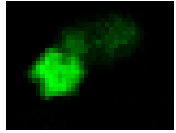

(b) Slice at $z=1$

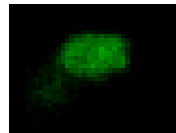

(c) Slice at $z=4$

Figure 7: An identical cell in MIP image and slices. All images are cropped following the same coordinates.

[12] Hyeonseob Nam and Bohyung Han. 2016. Learning Multi-domain Convolutional Neural Networks for Visual Tracking. In 2016 IEEE Conference on Computer Vision and Pattern Recognition (CVPR'16). 4293-4302.

[13] Mikael J. Pittet and Ralph Weissler. 2011. Intravital imaging. Cell 147, 5 (2011), 983-991.

[14] Caroline A. Schneider, Wayne S. Rasband, and Kevin W. Eliceiri. 2012. NIH Image to ImageJ: 25 years of image analysis. Nature methods 9, 7 (2012), 671-675.

[15] Hironori Shigeta, Shigeto Seno, Shino Nishizawa, Yutaka Uchida, Junichi Kikuta, Masaru Ishii, and Hideo Matsuda. 2019. Analyzing leukocyte migration trajectories by deformable image matching. In 2019 IEEE 19th International Conference on Bioinformatics and Bioengineering (BIBE). IEEE, 94-98.

[16] Hsieh-Fu Tsai, Joanna Gajda, Tyler FW Sloan, Andrei Rares, and Amy Q Shen. 2019. Usiigaci: Instance-aware cell tracking in stain-free phase contrast microscopy enabled by machine learning. SoftwareX 9 (2019), 230-237.

[17] Bo Wu and Ram Nevatia. 2006. Tracking of Multiple, Partially Occluded Humans based on Static Body Part Detection. In 2006 IEEE Computer Society Conference on Computer Vision and Pattern Recognition (CVPR'06), Vol. 1. 951-958.

[18] Xiaodong Yang, Houqiang Li, and Xiaobo Zhou. 2006. Nuclei Segmentation Using Marker-Controlled Watershed, Tracking Using Mean-Shift, and Kalman Filter in Time-Lapse Microscopy. IEEE Transactions on Circuits and Systems I: Regular Papers 53, 11 (2006), 2405-2414.

[19] Faliu Yi and Inkyu Moon. 2012. Image segmentation: A survey of graph-cut methods. In 2012 International Conference on Systems and Informatics (ICSAI2012). 1936-1941. 